\title{
Advancing cycling among women: An exploratory study of North American cyclists
}

\author{
Huyen T. K. Le \\ School of Public and International Affairs, \\ Virginia Tech \\ huyenle@vt.edu
}

Fionnuala Quinn

Discover Traffic Gardens

\author{
Alyson West \\ The University of North Carolina Highway \\ and Safety Research Center \\ Steve Hankey \\ School of Public and International Affairs \\ Virginia Tech
}

\begin{abstract}
Past studies show that women cycle at a lower rate than men due to various factors; few studies examine attitudes and perceptions of women cyclists on a large scale. This study aims to fill that gap by examining the cycling behaviors of women cyclists across multiple cities in North America. We analyzed an online survey of 1,868 women cyclists in the US and Canada, most of whom were confident when cycling. The survey recorded respondents' cycling skills, attitude, perceptions of safety, surrounding environment, and other factors that may affect the decision to bicycle for transport and recreation. We utilized tree-based machine learning methods (e.g., bagging, random forests, boosting) to select the most common motivations and concerns of these cyclists. Then we used chi-squared and non-parametric tests to examine the differences among cyclists of different skills and those who cycled for utilitarian and non-utilitarian purposes. Tree-based model results indicated that concerns about the lack of bicycle facilities, cycling culture, cycling's practicality, sustainability, and health were among the most important factors for women to cycle for transport or recreation. We found that very few cyclists cycled by necessity. Most cyclists, regardless of their comfort level, preferred cycling on facilities that were separated from vehicular traffic (e.g., separated bike lanes, trails). Our study suggests opportunities for designing healthy cities for women. Cities may enhance safety to increase cycling rates of women by tailoring policy prescriptions for cyclists of different skill groups who have different concerns. Strategies that were identified as beneficial across groups, such as investing in bicycle facilities and building a cycling culture in communities and at the workplace, could be useful to incorporate in long-range planning efforts.
\end{abstract}

Keywords: gender, perception, non-motorized transport, physical activity, active travel, machine learning

\section{Article history:}

Received: September 11, 2018

Received in revised form:

December 30, 2018

Accepted: April 11, 2019

Available online: May 15, 2019

Copyright 2019 Huyen T. K. Le, Alyson West, Fionnuala Quinn, \& Steve Hankey http://dx.doi.org/10.5198/jtlu.2019.1461

ISSN: $1938-7849$ | Licensed under the Creative Commons Attribution - Noncommercial License 4.0

The Journal of Transport and Land Use is the official journal of the World Society for Transport and Land Use (WSTLUR) and is published and sponsored by the University of Minnesota Center for Transportation Studies. 


\section{Introduction}

The fact that male cyclists outnumber female cyclists in North America is well documented (Garrard, Handy, \& Dill, 2012; Moudon et al., 2005; Pucher, Buehler, \& Seinen, 2011). Despite significant efforts to close this gap, for example, by leading and participating in women's rides, spurring conversation, passing on knowledge, and inspiring or mentoring beginner riders, the discrepancy remains. Numerous studies have examined travel behavior in an effort to understand which policies will advance cycling among women (Garrard, 2003; Garrard et al., 2012; Garrard, Rose, \& Lo, 2008). One common point of agreement is that in order to reduce the gender gap, we must first understand what drives women's cycling behavior, and then address certain audiences in a targeted fashion, not as a uniform whole.

A possible dimension to examine is by trip purpose, where recreational cyclists might have different needs and concerns as compared to those who cycle for transport. Women were consistently found to cycle for recreation more frequently than to cycle for utilitarian purposes (Aldred, 2016; Dill, 2009b; Grudgings et al., 2018). If the goal is to increase the amount of cycling among women, then it is essential to make utilitarian cycling a convenient and safe option for current recreational cyclists. Another important dimension is differences by skill or comfort level. The four types of cyclists (Geller, 2006) is a commonly used means of identifying different groups of cyclists by their own self-described individual attitudes towards cycling, also understood as their comfort level. Segmenting "cycling market" by skill or comfort level can assist in determining how to target different types of cyclists when planning to serve varying needs with infrastructure or promotional programming (Dill \& McNeil, 2016).

Despite the need for a better understanding of gender and cycling behavior, very few studies have specifically focused on women cyclists across multiple jurisdictions. This study aims to fill this gap by looking at behavior and perceptions of women cyclists in multiple cities across North America. We aim to answer several research questions related to women cycling rates: (1) What are the major motivators and barriers to cycling associated with utilitarian and non-utilitarian women cyclists; (2) Do skilled women cyclists cycle more for utilitarian purposes than cyclists from other skill groups; and (3) Do women cyclists with different skillsets perceive motivators and barriers to cycling (e.g., weather, terrain, safety concerns) differently? Exploring these questions contributes to the body of knowledge on gender and cycling. This work also assesses potential strategies and policies to advance cycling among women. It is noteworthy that due to our sample characteristics (i.e., highly skewed toward certain geographical areas and confident women cyclists), we do not explore the gender difference in cycling (i.e., between men and women cyclists) or generalize the results outside this particular group.

\section{Background}

Several studies have examined the topic of women and cycling with the goal of reducing the gender gap in cycling rates. Results from the 2010 Women Cycling Survey distributed by the Association of Pedestrian and Bicycle Professionals (APBP) showed that safety and infrastructure were the central concerns of women who bicycle, and that community size played a role in the cyclists' behavior patterns, which are consistent with findings from other studies (Dill \& McNeil, 2013; Sibley, 2010).

Studies comparing male and female riders suggest that women and men face similar barriers to cycling. Australian researchers found that men and women both prefer designated bike lanes or offroad paths when given the option (Heesch, Sahlqvist, \& Garrard, 2012). A study looking at university commuter cyclists in Columbus, $\mathrm{OH}$, found that, in general, stated barriers to cycling were very similar among women and men (Akar, Fischer, \& Namgung, 2013). However, another university campus study in Maryland showed that women were more sensitive to infrastructure and the environment (e.g., weather) (Abasahl, Kelarestaghi, \& Ermagun, 2018). 
Trip complexity is also a barrier to women cycling. Multiple studies found that women make different trip choices than men for utilitarian travel. For example, women tended to trip-chain more often and had more complex travel patterns (e.g., more trips, diverse trip purposes) (Heesch et al., 2012; McGuckin, Zmud, \& Nakamoto, 2005). Women's complex cycling trip patterns were considered a significant barrier to choosing to ride a bicycle (Bonham \& Wilson, 2012).

A common conclusion among studies is that to increase the number of women who cycle, we must design planning and policy strategies to target different groups of women who have varying comfort levels and needs. Emond, Tang, and Handy (2009) emphasized the need for gender sensitivity in planning and suggests targeting "experienced" and "less-experienced" cyclists separately. Several studies have explored methods for classifying cyclists. Damant-Sirois and El-Geneidy (2015) surveyed cyclists in Montréal and divided the respondents into four determinant categories based on a combination of individual characteristics and attitudes, local cultural norms, and the built environment. Dill and McNeil (2016) worked with Oregon travel survey data to revisit the four types of cyclists as posited by Geller (2006). The study found that despite the limitations, the comfort level classification was a reasonable, very broad method of dividing cyclists into groups.

The body of literature suggests that increasing safety and connectivity in cycling networks will aid in encouraging more women to cycle. Dill tracked 160 cyclists in Portland and studied revealed route preferences based on their travel patterns. The study showed a need for improving connections in cycling infrastructure (Dill, 2009a). Moudon et al (2005) found that infrastructure connecting cyclists to transit positively affected a person's likelihood to cycle. Teschke et al.'s study on cyclists in Montréal and Vancouver showed that cycling mode share increased greatly where bicycle infrastructure was best, and the gender gap in those locations was largely reduced (Teschke, Chinn, \& Brauer, 2017).

Additional studies show that culture and encouragement is a powerful tool to increase cycling among women. Singleton and Goddard (2016) analyzed the cycling gender gap in Oregon travel survey data from a socioeconomic perspective and posited that women's ride groups such as "We Bike New York" could help encourage more women to cycle. Another study found that where cycling is perceived as normal within the community, more people tend to cycle (Xing, Handy, \& Mokhtarian, 2010). Perception of safety, which influences whether people cycle, can be improved through programming such as Open Streets and with wayfinding. Giving new riders a chance to feel more comfortable on the streets may contribute to more women riding (Ma, Dill, \& Mohr, 2014).

When surveying cyclists' concerns and perceptions, researchers usually applied Likert scale or binary responses. While this is an efficient way to obtain responses from participants, such measurement poses several difficulties as discussed in the following Methodology section. Traditional multivariate statistical methods were usually employed, such as Pearson's chi-squared test, ANOVA (Dill \& McNeil, 2016; Heesch et al., 2012), and logit models (Abasahl et al., 2018; Garrard et al., 2008), among other methods. Non-parametric methods have rarely been applied. Further, new methods, such as machine learning, have been developed and widely applied in transportation research. However, to the best of our knowledge, no cycling survey studies have used these methods. Machine learning methods have been commonly used for prediction or pattern recognition (Arentze \& Timmermans, 2004; Gong, Kanamori, \& Yamamoto, 2017; Gong, Morikawa, Yamamoto, \& Sato, 2014; Jahangiri \& Rakha, 2015), traffic and crash analysis (Antoniou \& Koutsopoulos, 2006; Ding, Zhao, \& Jiao, 2002), as well as smartphone and GPS data (Arentze \& Timmermans, 2004; Gong et al., 2017, 2014; Jahangiri \& Rakha, 2015). Few studies have employed machine learning to analyze survey data (Ding, Cao, \& Næss, 2018; Varian, 2014) as an alternative method to traditional statistical methods for identifying patterns among survey responses. 


\section{$3 \quad$ Methodology}

\subsection{Data collection}

The Women Cycling study was conducted in the spring of 2016 by the APBP and co-led by one of the authors. Participants were asked to report their socio-demographic information as well as their cyclingrelated perceptions and concerns. The survey was administered online through SurveyMonkey and took about 15 minutes to complete. Participants received no incentives upon completion. The study was open to anyone who identified as a woman or girl and was interested in cycling. The survey was distributed through social media, advocacy groups for cyclists, and requests to national cycling and health organizations.

In total, 1,896 respondents completed the survey. As a result of the recruitment methods, the sample was dominated by women cyclists who lived and worked in the US and Canada (Figure 1). To increase homogeneity, respondents who were not women and did not live in North America were dropped from our analyses. The final data set consisted of 1,868 participants.

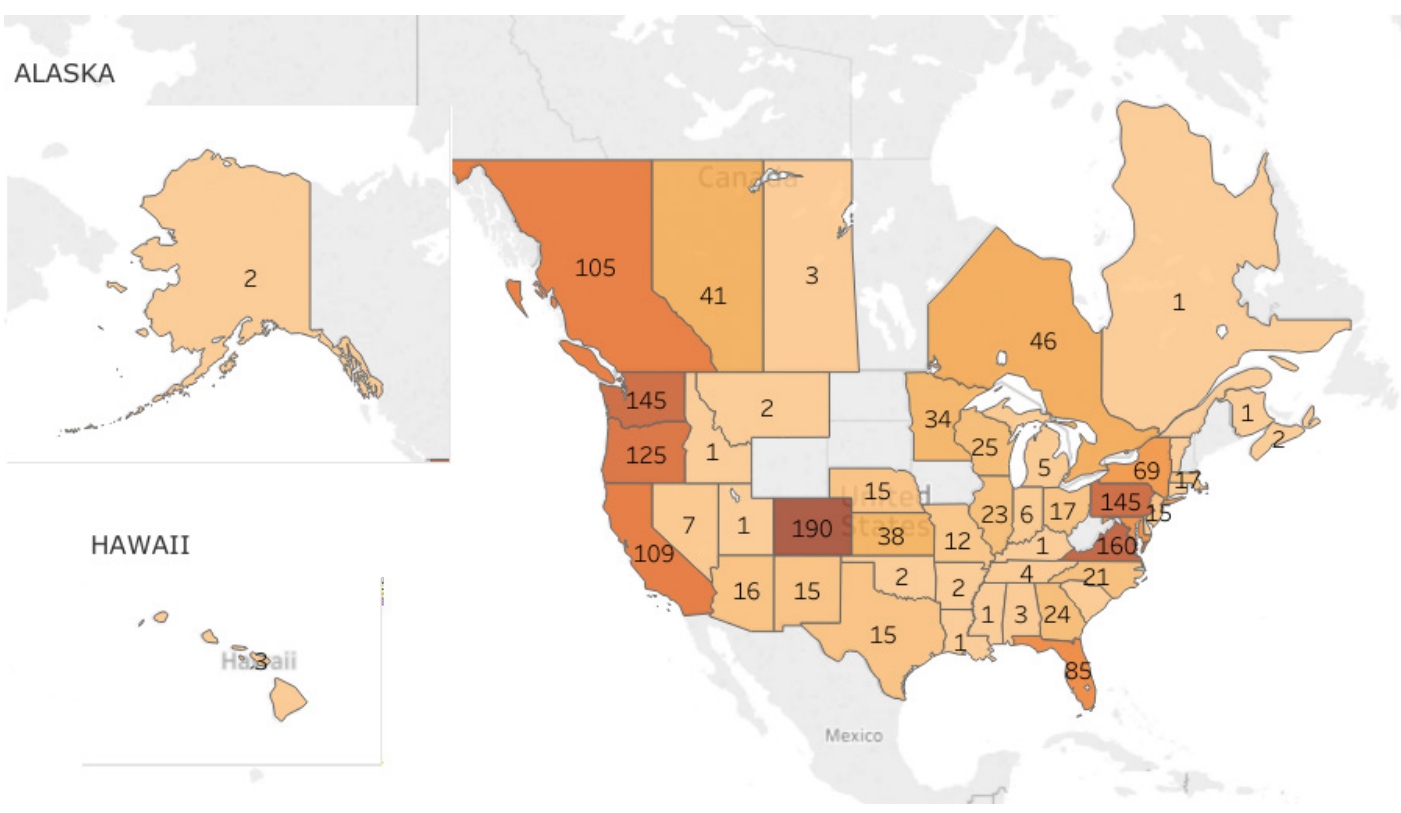

Figure 1. Distribution of the Women Cycling Survey sample by state or province

\subsection{Variable measurement}

The survey mainly consisted of multiple-choice questions with single or multiple responses. Participants were asked to report their cycling usage, such as destinations and trip purpose that were made by bicycle, the reasons that they chose to cycle, type of bicycle (e.g., road bike, cargo, bikeshare, mountain bike), as well as the minimum and ideal bicycle facilities required in order to make them feel comfortable when cycling. Additionally, the survey included attitudinal questions where respondents were asked to rate the importance of various factors that affect their bicycling patterns such as traffic safety, facilities, obstacles to cycling, and education and policies related to promoting cycling. The answers were provided on a 5-point Likert scale from 1 (Not at all Important) to 5 (Very Important). Participants also self-identified their skill level based on Geller's (2006) definition of the four types of cyclists. The question reads: "What general category of transportation bicyclist would you mostly place yourself in (i.e. comfort level using available facilities)?” Respondents were presented with four options: Strong and Fearless (cycle regardless 
of road condition), Enthused and Confident (comfortable sharing road but prefer own facilities), Interested but Concerned (curious about cycling, would ride iffelt safer on road), and No Way No How (not interested in cycling at all).

We recoded the variables for the modeling work and non-parametric tests. For example, we grouped bicycle facility into two types: "on-street" consists of bicycle facilities with pavement markings (e.g., bike boulevards, bike lanes, buffered bike lanes), and "off-street" consists of off-street trails, sidewalks, and shared-use paths. Participants were also able to choose "I would bike regardless, $t$ " indicating no infrastructure was needed in order to encourage them to cycle, thus we coded this type as "anything." Participants were able to select multiple reasons for their cycling choice. We coded the responses into multiple binary variables, with " 1 " indicating that the reason was selected. The full list of variables is shown in Table 3.

\subsection{Analysis}

We first used exploratory analyses of descriptive statistics to assess responses. We then employed several machine learning models to explore important factors perceived by cyclists who cycled for utilitarian purposes (i.e., transport) and non-utilitarian purposes (i.e., recreation). Finally, we performed several hypothesis tests on the important variables identified in the machine learning models to explore those factors in detail.

Machine learning is a set of approaches that automate model building and learn from the data (i.e., learns the relationship between the predictors and outcomes to make predictions) (Hastie, Tibshirani, \& Friedman, 2009). It has been used widely in transportation to accomplish robust prediction and pattern recognition objectives. The latter purpose (pattern recognition) has been rarely applied to transportation survey data, as transportation surveys usually have relatively small sample sizes, and/or traditional statistical and econometric methods are adequate to answer the research questions. For some purposes, machine learning methods (e.g., tree-based models) would be a better option as they are able to handle a large number of nominal variables - which is the case in our study where continuous variables were unavailable. The data reported here may not be suitable for traditional regression methods as multicollinearity is very likely to occur, increasing the potential for Type 2 error (i.e., failing to reject the null hypothesis when it is true). The machine learning models also work well with nonlinearity and interaction effects and could be a good alternative or even outperform econometric methods when analyzing survey data in some cases (Varian, 2014).

Tree-based methods are a family of models that involves growing decision trees (Hastie et al, 2009; James, Whitten, Hastie, \& Tibshirani, 2014). The process of growing trees involves stratifying or segmenting the predictor space into various regions, then using the mode of the training observations in its own region (Hastie et al., 2009; James et al., 2014). As such, tree methods can determine which variables are important in prediction (i.e., account for most variance in the data) and identify which predictors best improve prediction accuracy (James et al., 2014; Varian, 2014).

Decision tree results are often very straightforward as it mimics the human decision process. However, growing a single tree would result in overfitting (i.e., the tree is very sensitive to the input data), thus it has limited out-of-sample prediction and inference capability. Therefore, other tree-based methods were developed to overcome this limitation by repeatedly growing trees from subsamples of the training sets. The probability that a variable is selected in the tree is averaged (or the majority vote is taken) to report the final results. However, results from these models are now the summary of many iterations of the model/tree growth, and thus are more difficult to identify directions of effect. In other words, these results only identify the important variables; thus, we must rely on other methods to determine the direction of effect for each variable shown in the models. 
In our study, tree-based methods such as bagging, random forests, and boosting were applied to recognize patterns in cycling by trip purpose. Bagging (Breiman, 1996) is a method of repeatedly sampling the data (i.e., bootstrapping) and creating multiple tree models, then aggregating the tree model results to reduce the variance and increase prediction accuracy. Random forests (Breiman, 2001) operates on the same principle, except that it improves bagging by decorrelating trees: each time a tree model is built, only a subset of fresh predictors is considered at each split. The trees are then averaged. Boosting (Bühlmann \& Hothorn, 2007), on the other hand, grows trees sequentially instead of bootstrapping, such that each new tree is grown using the information of the previous one. We used all three methods and performed 10 -fold cross validation by randomly splitting the dataset into 10 equal folds, training the model with $90 \%$ of the sample data and testing its performance on the remaining $10 \%$ of the data. This procedure was performed on 5,000 trees and 99 iterations of 10-fold cross validation to avoid overfitting and to check the robustness of the models. We used the randomForest and $g b m$ packages in $\mathrm{R}$ for these analyses.

Since our study aims to explore the important factors cited by cyclists when they cycled for each purpose (i.e., utilitarian or non-utilitarian purpose), we developed two sets of models for each trip purpose. The outcome variables are binary (cycle for utilitarian/non-utilitarian purpose or not). We included all socio-demographic variables along with responses to the attitudinal question in the models. We expect that these factors differ among groups of cyclists with different skillsets or comfort levels. Ideally this analysis would also be performed for each group; however, splitting into skill groups would reduce the sample size, which results in less-robust models. We performed statistical tests on pooled data from three of the groups ( $\mathrm{n}=1,762$; Interested but Concerned, Enthused and Confident, and Strong and Fearless). We excluded the No Way No How group from all analyses due to its small sample size $(\mathrm{n}=12)$.

Based on the list of most important variables from the machine learning models, we conducted further statistical tests to examine the direction of effect for key variables in the data. We chose to only test the most important variables identified in the machine learning models, instead of testing all the variables in the dataset, in order to reduce Type I error (i.e., Type I error increases when multiple significance tests for too many variables are conducted on the same sample). Given that most variables in the dataset were measured at the nominal or ordinal scale, we performed statistical tests as appropriate: chi-squared, Kruskal-Wallis, and Wilcoxon rank-sum. The Kruskal-Wallis (KW) test is a non-parametric test that is equivalent to the one-way ANOVA parametric test when the distribution is non-normal or unknown, while the Wilcoxon rank-sum test is a non-parametric counterpart of the independent t-test. Both Kruskal-Wallis and Wilcoxon rank-sum tests hypothesize that the independent groups are drawn from the same or different distribution(s). While the Kruskal-Wallis test only shows whether at least one group is different from all other groups, it does not show the direction of effect. We performed a series of post-hoc rank-sum tests and applied Bonferroni correction to account for inflated Type 1 error. The analyses were performed in Stata IC 14.2.

\section{$4 \quad$ Results}

This section provides some descriptive results from the survey, followed by the machine learning models, chi-squared, Kruskal-Wallis, and Wilcoxon rank-sum tests.

\subsection{Descriptive statistics}

The sample consisted of 1,868 respondents from 52 states and provinces (Figure 1). A large number of participants came from Colorado, Pennsylvania, Virginia, Washington, Oregon, California, and British Columbia. Several less populated states and provinces did not have any participants, such as Wyoming, 
North Dakota, South Dakota, Iowa, and West Virginia, among others.

A large proportion of our sample is aged 30-39, and roughly equal groups of women in their 20s, 40s, and 50s (Figure 2). The sample is dominated by highly educated respondents who completed college or graduate school. Eighty seven percent of respondents were white. Seventy three percent of the respondents had no school-aged children.

\section{AGE}

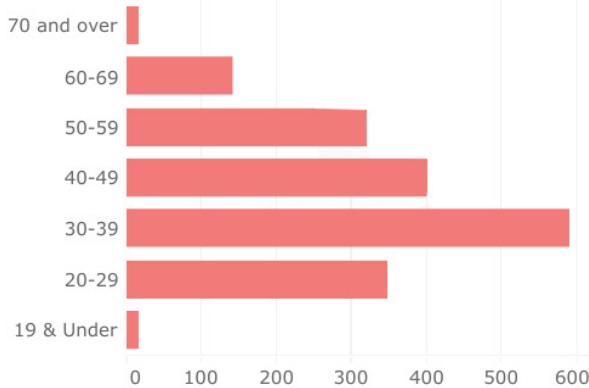

\section{EDUCATION}

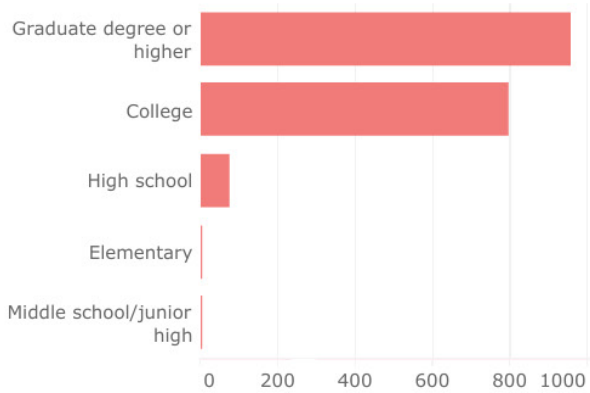

\section{RACE}

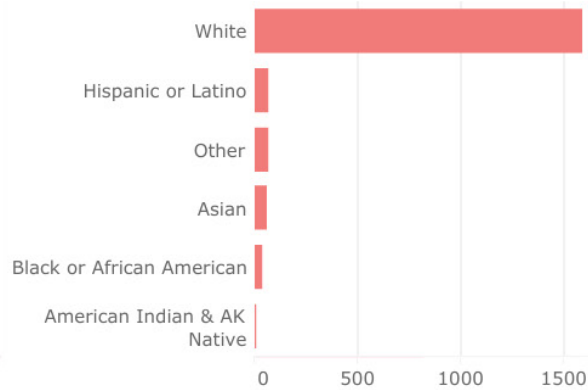

Figure 2. Socio-demographics of the Women Cycling Survey respondents

With regards to skill level, Table 1 shows the composition of our sample as compared to the proportional representations of each skill group (or comfort level group) based on a random sample of 3,000 respondents across the US (Dill \& McNeil, 2016). Since the survey targeted women cyclists, we oversampled the first two skill groups (Strong and Fearless and Enthused and Confident), and under-sampled the other two groups.

Table 1. Comparison of the WCS sample to Dill and McNeil (2016)

\begin{tabular}{lrrrr}
\hline Skill levels & National (All) & National (Men) & National (Women) & This study \\
\hline Strong and Fearless & $7 \%$ & $8 \%$ & $6 \%$ & $22 \%$ \\
Enthused and Confident & $5 \%$ & $7 \%$ & $3 \%$ & $61 \%$ \\
Interested but Concerned & $51 \%$ & $55 \%$ & $47 \%$ & $17 \%$ \\
No Way, No How & $37 \%$ & $29 \%$ & $44 \%$ & $<1 \%$ \\
\hline
\end{tabular}

Table 2 shows the socio-demographic distribution across the three large groups in our sample (excluding No Way No How). Overall, the sample is highly skewed toward Enthused and Confident cyclists in all categories. The other two groups are roughly equal in all categories except age and cycling purpose, although these discrepancies are not substantial. For example, more Interested but Concerned cyclists are below 19 or above 60 years old, and fewer of them cycled for either utilitarian or non-utilitarian purpose. 
Table 2. Socio-demographic distribution across three types of cyclists in our sample (Column percentage shown in parentheses)

\begin{tabular}{|c|c|c|c|c|}
\hline & $\begin{array}{r}\text { Interested but } \\
\text { Concerned }\end{array}$ & $\begin{array}{r}\text { Enthused and } \\
\text { Confident }\end{array}$ & $\begin{array}{r}\text { Strong and } \\
\text { Fearless }\end{array}$ & Total \\
\hline Age & 296 & 1,081 & 385 & 1,762 \\
\hline $19 \&$ Under & $8(3 \%)$ & $1(0.1 \%)$ & $2(1 \%)$ & $11(1 \%)$ \\
\hline $20-29$ & $59(20 \%)$ & $192(18 \%)$ & $79(21 \%)$ & $330(19 \%)$ \\
\hline $30-39$ & $68(23 \%)$ & $362(33 \%)$ & $146(38 \%)$ & $576(33 \%)$ \\
\hline $40-49$ & $79(27 \%)$ & $231(21 \%)$ & $78(20 \%)$ & $388(22 \%)$ \\
\hline $50-59$ & $50(17 \%)$ & $197(18 \%)$ & $60(16 \%)$ & $307(17 \%)$ \\
\hline $60-69$ & $27(9 \%)$ & $90(8 \%)$ & $19(5 \%)$ & $136(8 \%)$ \\
\hline 70 and over & $5(2 \%)$ & $8(1 \%)$ & $1(0.3 \%)$ & $14(1 \%)$ \\
\hline Education & 295 & 1,077 & 385 & 1,757 \\
\hline College & $137(46 \%)$ & $477(44 \%)$ & $149(39 \%)$ & $763(43 \%)$ \\
\hline Elementary & $0(0 \%)$ & $0(0 \%)$ & $1(0.3 \%)$ & $1(0.1 \%)$ \\
\hline Graduate degree or higher & $143(48 \%)$ & $567(53 \%)$ & $218(57 \%)$ & $928(53 \%)$ \\
\hline High school & $15(5 \%)$ & $33(3 \%)$ & $16(4 \%)$ & $64(4 \%)$ \\
\hline Middle schooljjunior & $0(0 \%)$ & $0(0 \%)$ & $1(0.3 \%)$ & $1(0.1 \%)$ \\
\hline Race & 294 & 1,075 & 384 & 1,753 \\
\hline American Indian \& AK Native & $0(0 \%)$ & $6(1 \%)$ & $2(1 \%)$ & $8(0.5 \%)$ \\
\hline Asian & $14(5 \%)$ & $29(3 \%)$ & $12(3 \%)$ & $55(3 \%)$ \\
\hline Black or African American & $7(2 \%)$ & $21(2 \%)$ & $7(2 \%)$ & $35(2 \%)$ \\
\hline Hispanic or Latino & $15(5 \%)$ & $36(3 \%)$ & $11(3 \%)$ & $62(4 \%)$ \\
\hline Other & $15(5 \%)$ & $36(3 \%)$ & $11(3 \%)$ & $62(4 \%)$ \\
\hline White & $243(83 \%)$ & $947(88 \%)$ & $341(89 \%)$ & $1,531(87 \%)$ \\
\hline Having young children & $80(27 \%)$ & $266(25 \%)$ & $67(17 \%)$ & $413(23 \%)$ \\
\hline Cycling for utilitarian purpose & $106(36 \%)$ & $903(84 \%)$ & $354(92 \%)$ & $1,363(77 \%)$ \\
\hline Cycling for non-utilitarian purpose & $232(78 \%)$ & $1,003(93 \%)$ & $368(96 \%)$ & $1,603(91 \%)$ \\
\hline
\end{tabular}

Figure 3 shows the responses with regard to ideal bicycle facilities and minimum facilities for cyclists to start cycling. The graphs distinguish different skill levels of cyclists in our sample. The percentage indicates the proportion of cyclists in each group for each type of facility. Although many cyclists reported that they would cycle using on-street facilities or places without a facility, a sizable number of cyclists indicated that they would only cycle on bike lanes. Notably, a majority of the Interested but Concerned group indicated their minimum facilities as bike lanes, followed by buffered bike lanes, and on-street. When it comes to ideal types of facility, most cyclists reported that they preferred cycling on trails and buffered bike lanes regardless of their comfort level.

Women cycled for different purposes and reasons (Figure 4). The percentage represents the proportion of respondents in each skill level who cycled for the corresponding purpose (each participant selected one or more responses). Most participants cycled for exercise and recreation. Cycling for transportation (including commute to work) was also common but to a lesser degree. Most respondents indicated that the main reasons for them to cycle, in descending order, were health, joy, practicality, sustainability, and affordability. Very few respondents chose to cycle because it was their only option, however, affordability was one of the main reasons for cycling. 


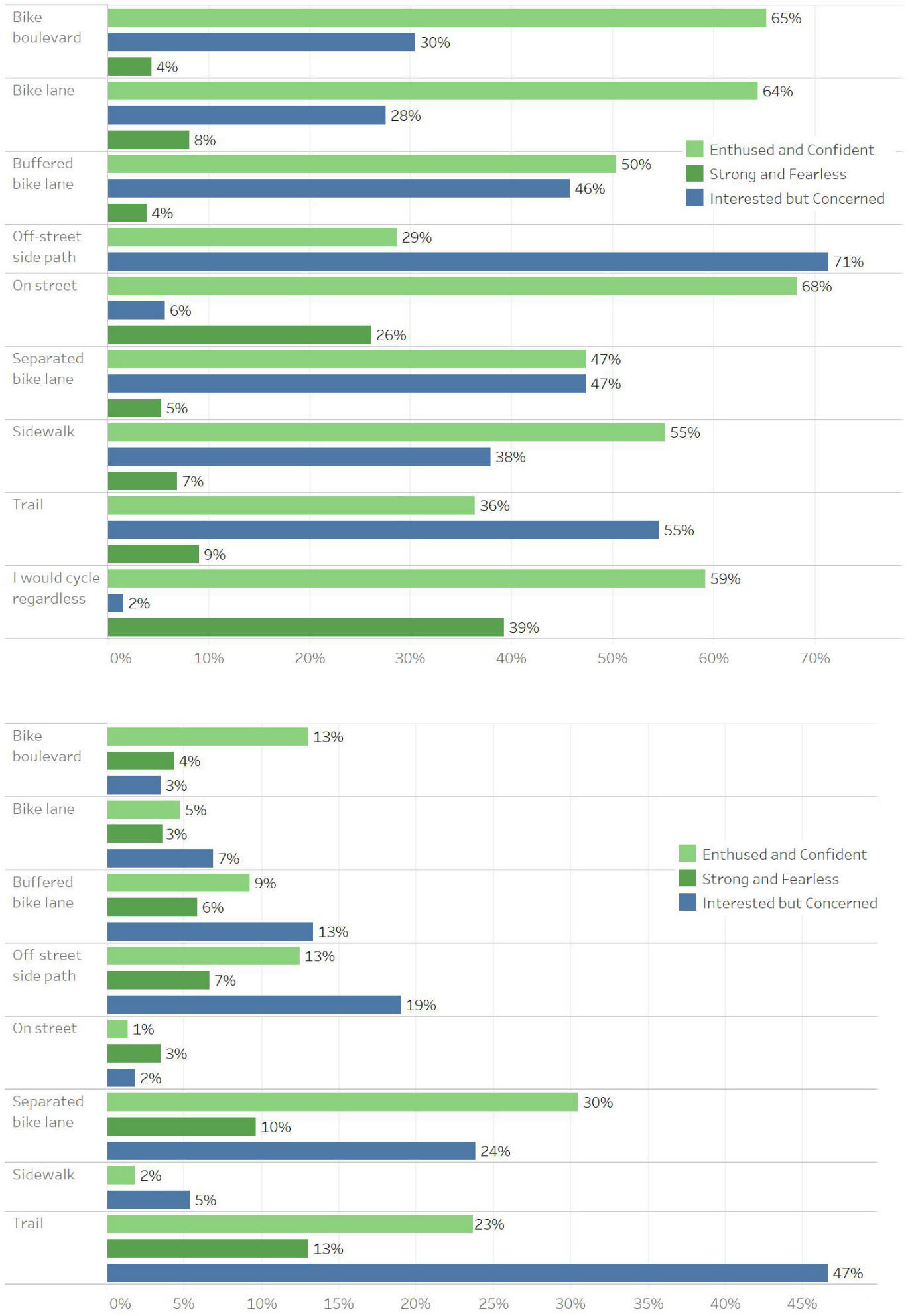

Figure 3. Distributions of responses for questions on minimum (top chart) and ideal bicycle facility (bottom chart) 

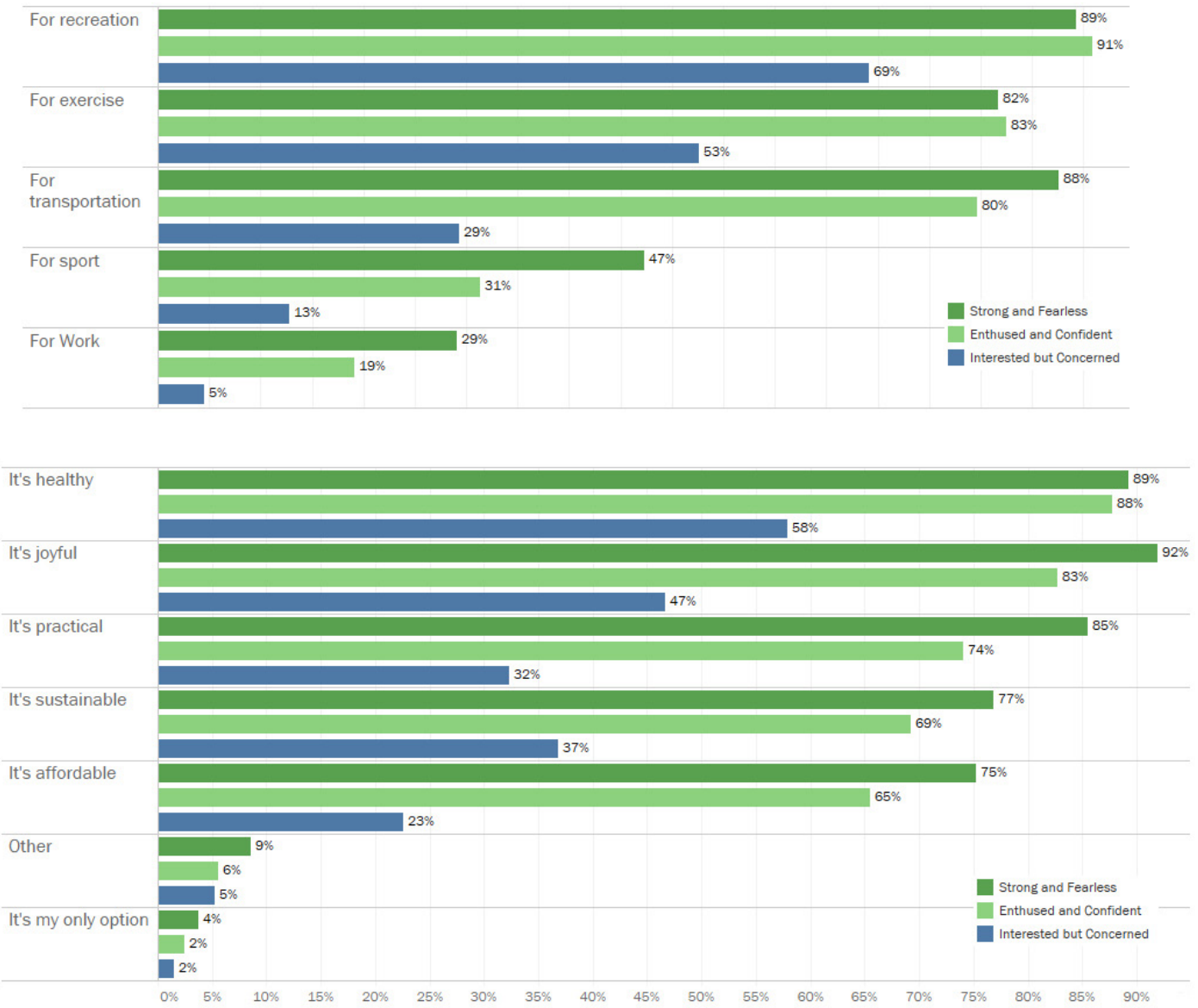

Figure 4. Distributions of responses for questions on trip purpose (top chart), and reasons for cycling (bottom chart)

Table 3 describes other variables that were measured in our data set. The indicators include safety concerns, features of facilities, desired changes in culture to promote cycling, and obstacles to cycling. Each respondent rated the importance of each factor by choosing a value from 1 (Not at All Important) to 5 (Very Important). 
Table 3. Descriptive statistics of the Women Cycling Survey responses

\begin{tabular}{|c|c|c|c|}
\hline Variable & Percent & Mean & Std. Dev. \\
\hline No school-aged children & 72.86 & & \\
\hline Safety: Reduced traffic speeds & & 3.84 & 1.18 \\
\hline Safety: More aware drivers & & 4.62 & 0.76 \\
\hline Safety: Fewer vehicles & & 3.79 & 1.11 \\
\hline Safety: Lighting & & 3.58 & 1.15 \\
\hline Safety: Police presence & & 2.61 & 1.20 \\
\hline Safety: Ice/snow/leaves/debris cleared from cycling infrastructure & & 4.01 & 1.09 \\
\hline Safety: Road/path surface is in good condition & & 4.23 & 0.91 \\
\hline Facility: Safer places to cycle on roads & & 4.52 & 0.80 \\
\hline Facility: Better connectivity/more direct routes & & 4.54 & 0.79 \\
\hline Facility: Elimination of dangerous/unpleasant bottleneck & & 4.19 & 0.98 \\
\hline Facility: Installing wayfinding signs & & 3.58 & 1.19 \\
\hline Facility: Convenient bicycle parking & & 3.70 & 1.11 \\
\hline Facility: Bike lanes or bike paths connecting to desired transit stop/station & & 3.80 & 1.21 \\
\hline Culture: Community that embraces cycling & & 4.20 & 0.98 \\
\hline Culture: Workplace that embraces cycling & & 3.98 & 1.09 \\
\hline Culture: School/university that embraces cycling & & 3.79 & 1.22 \\
\hline Culture: More people cycling/greater cultural acceptance & & 4.08 & 1.09 \\
\hline Culture: A place to freshen up a little at my work & & 3.83 & 1.22 \\
\hline Culture: A place to change clothing $\&$ shower at my work & & 3.70 & 1.31 \\
\hline Culture: Financial incentives & & 3.17 & 1.44 \\
\hline Negative factor: Rain & & 3.39 & 1.29 \\
\hline Negative factor: Snow & & 4.21 & 1.16 \\
\hline Negative factor: Cold weather & & 2.96 & 1.29 \\
\hline Negative factor: Hot weather & & 2.39 & 1.20 \\
\hline Negative factor: Hills & & 2.47 & 1.21 \\
\hline Negative factor: personal safety & & 3.95 & 1.17 \\
\hline Negative factor: Environmental (air quality, sun exposure) & & 2.81 & 1.20 \\
\hline
\end{tabular}

Note: All variables, except "no school-aged children," range from 1-5.

\subsection{Important motivators and barriers to women's utilitarian and non-utilitarian cycling}

We developed models using random forests, bagging, and boosting to examine the patterns of cycling by trip purpose. Ten-fold cross-validation results showed a high out-of-sample prediction rate $(87 \%$ for random forests and bagging; $90 \%$ for boosting). Since the bagging results were very similar to the results from random forests, we only discuss the random forests and boosting results in this section.

Figure 5 shows the 20 most important factors for cyclists who cycled for utilitarian purposes. The importance of a variable indicates the contribution of this variable to the model's predictive power (i.e., the increase in prediction error when the variable is excluded). The higher the importance, the more relevant the variable is in predicting the outcome (e.g., cycling purpose). The models show that practicality is the most important factor associated with women's bicycling, followed by sustainability, affordability, and joy. Cycling skill (comfort level) and types of minimum (acceptable) facility are also important factors, although the direction of effect is unclear with this model. Other important factors are age, environmental exposure (e.g., sunlight, pollution), weather (e.g., cold or hot weather, snow, rain), the presence of police, wayfinding, and bottleneck traffic, among others. 


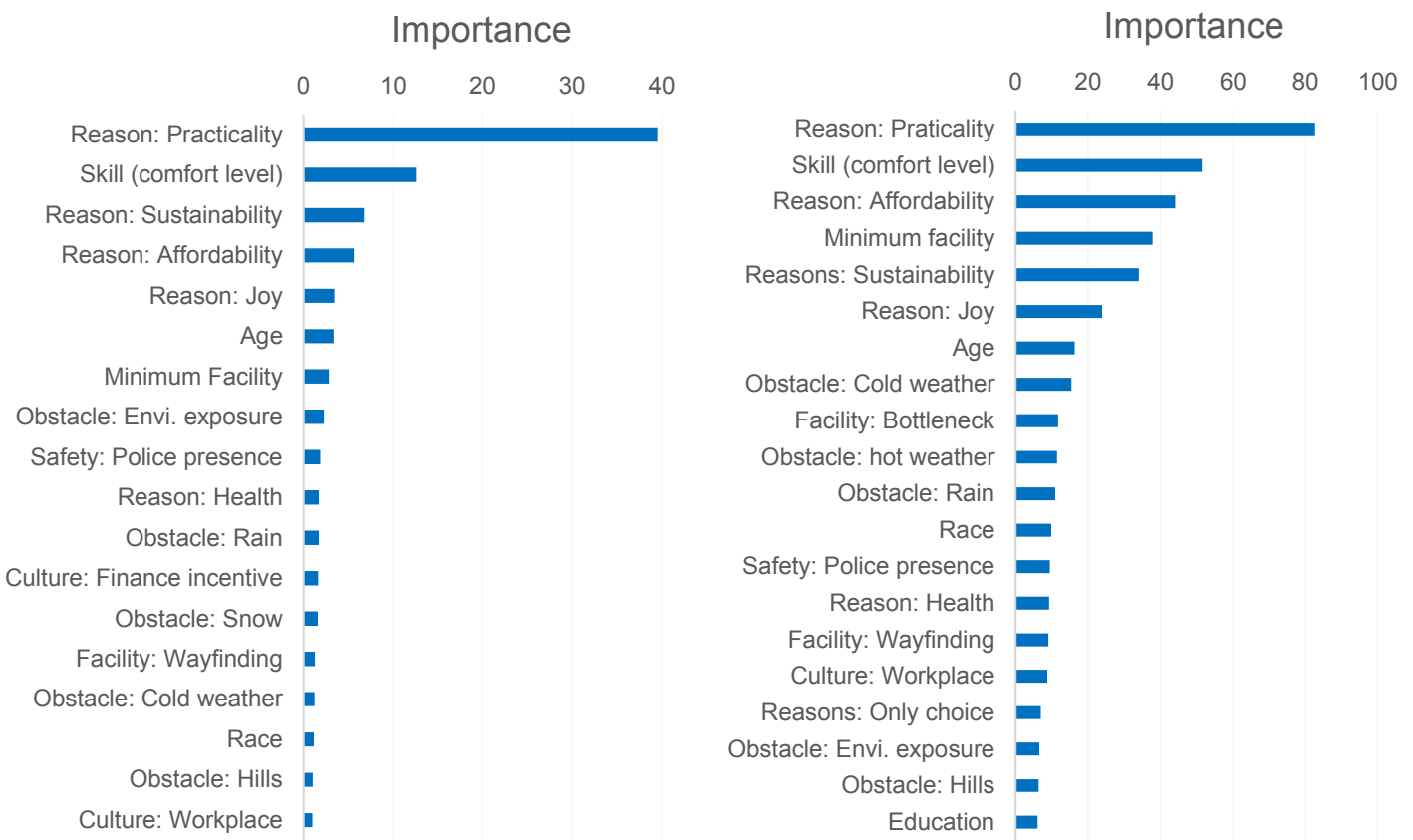

Figure 5. Results from the boosting (left) and random forests (right) models for utilitarian cycling

Figure 6 shows 20 variables that account for the most variance in the non-utilitarian model. For the non-utilitarian purposes, the most important reasons to cycle are joy and health. Comfort level (cycling skills) was also a factor, along with age, weather, police presence, and bicycle culture within the community or at work, among others.

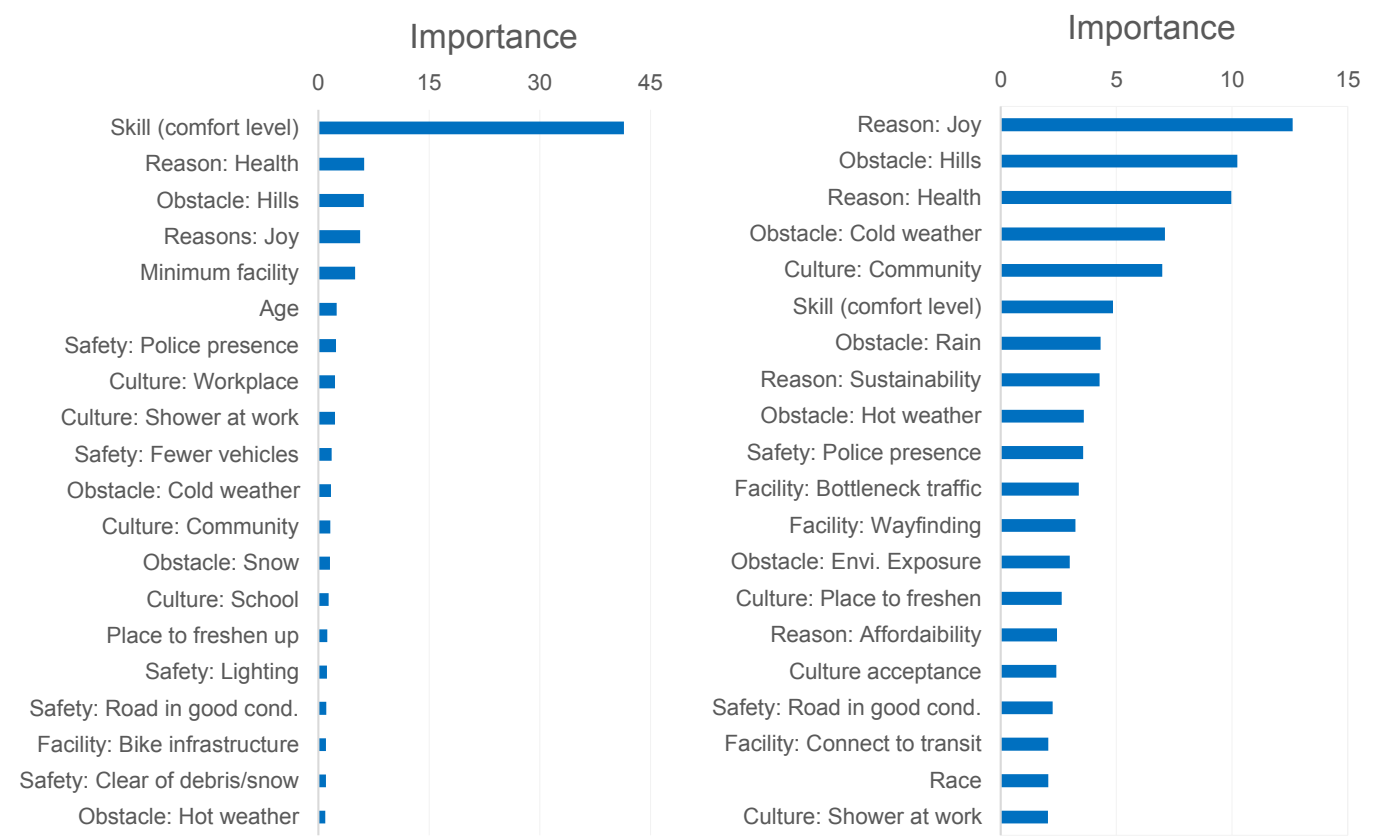

Figure 6. Results from the boosting (left) and random forests (right) models for non-utilitarian cycling 


\subsection{Promoting cycling based on skill groups}

Given that the above models did not show a consistent pattern for each variable among skill groups, we explored the differences in cyclists' behavior and perceptions by (1) cycling purpose and (2) groups of cyclists with different skill (or comfort level) based on the common variables in the above models. Table 4 shows the results from chi-squared, Wilcoxon rank-sum, and Kruskal-Wallis tests for utilitarian cycling (binary), non-utilitarian cycling (binary), and skill groups (4 groups). The percentages in the table were calculated as the proportions of participants in each group that fall into the corresponding categories and thus the row sum exceeds 100\%. For example, 99.7\% of participants in the Strong and Fearless group cited health as a motivation to cycle. We tested the hypothesis that there are no differences among groups for each motivator or barrier. The tested groups, shown in Table 4, are (1) utilitarian cyclists vs. other, (2) non-utilitarian cyclists vs. other, and (3) the three groups of cyclists by skill level (excluding the No Way No How group).

Most statistical tests for trip purpose were significant at the $\mathrm{p}<0.05$ cut-off level. Utilitarian cycling was different by age, but non-utilitarian cycling was not. Cycling culture was cited to be the only important factor for those who cycled for non-utilitarian purposes, whereas culture at the workplace was considered important for utilitarian cycling. Police presence was important for those who did not cycle for transport or recreation. Inclement weather only mattered to utilitarian cyclists. Hills were significant, although the sign of the test indicates that recreational cyclists were less likely to consider hills obstacles. 
Table 4. Results of the statistical tests on important factors identified in the machine learning models

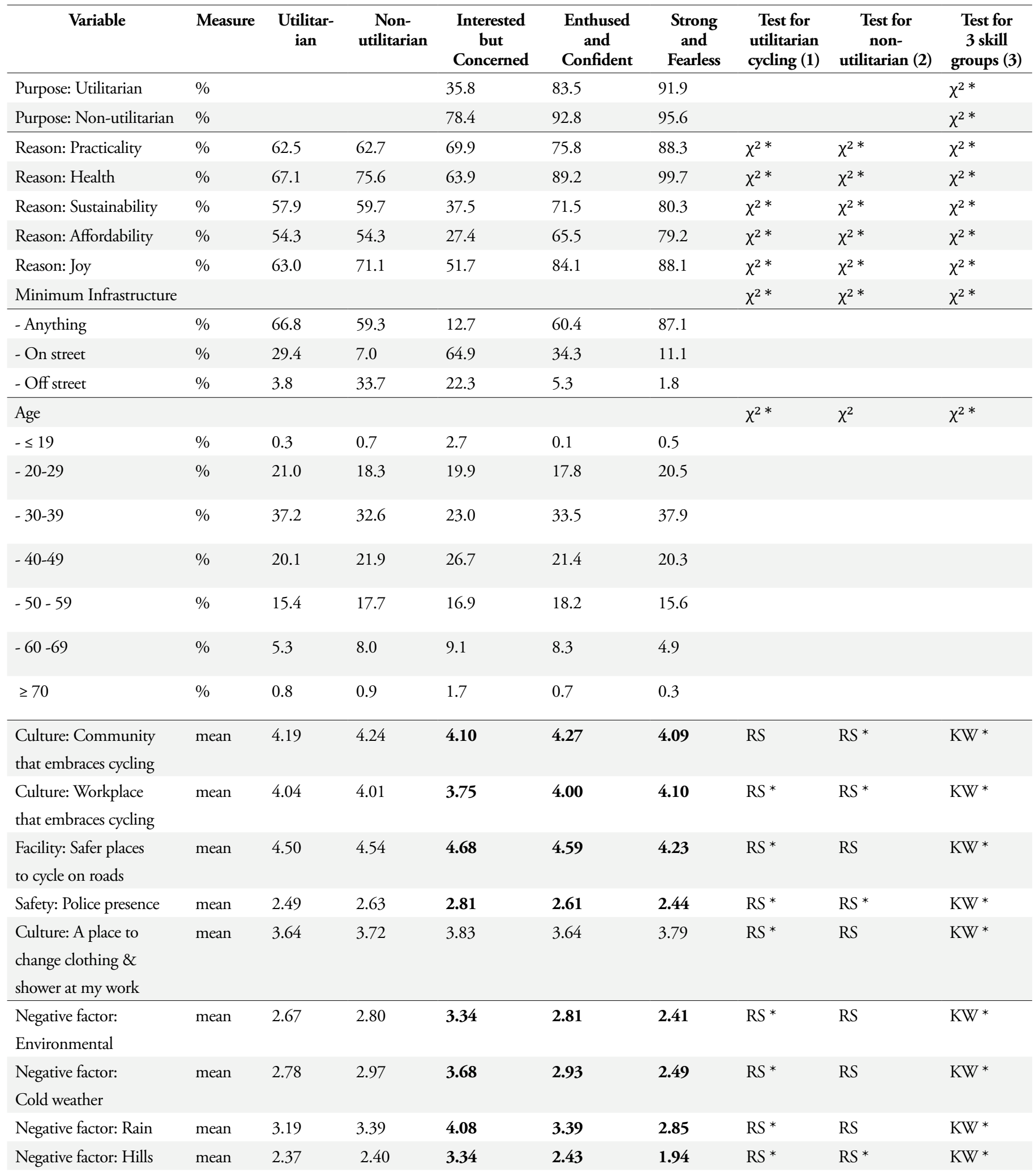

Note: Percentage shows the percent of respondents within each group that fall into the corresponding category. $\left(^{*}\right)$ denotes $p<0.05$. Test abbreviations: RS: Rank-sum, KW: Kruskal-Wallis. Values shown in bold text are significant at $p<0.05$ in post-hoc rank-sum tests (after Kruskal-Wallis tests) with Bonferroni correction. 
We also ran the statistical tests for the three groups of cyclists by skill level (Table 4). All chi-squared and Kruskal-Wallis tests were significant at $p<0.05$. We performed post-hoc rank-sum tests after each Kruskal-Wallis test to examine the direction of effect; significant results are shown in bold text in Table 4. The results suggest heterogeneity among groups based on skill levels; in other words, cyclists with different skill levels perceived the importance of these promotional strategies differently. A smaller percentage of respondents in the Interested but Concerned group cycled for utilitarian purposes (36\%), whereas a large majority of the high-skilled cyclist groups cycled for utilitarian purposes. Consistent with the name, the No Way No How group did not participate in cycling as much, even for non-utilitarian purposes (we did not include this group in our analyses).

With regards to the reasons for cycling, all three groups of higher-skilled cyclists cited practicality and health (Table 4). Affordability was cited less often, while joy and sustainability seemed to be a big motivation for the last two groups. Notably, although sustainability was cited less often in the Interested but Concerned group, it was still the main reason for them to try cycling occasionally.

With respect to motivators to cycling, Enthused and Confident cyclists felt a higher need for a community cycling culture than other groups. The Strong and Fearless group, on the other hand, emphasized the importance of a cycling culture at the workplace at a higher rate than other groups. Facility was more likely to be cited as a motivator by the Interested but Concerned group, followed by the Enthused and Confident group. Police presence was more important to the lower skilled groups. Similarly, the lower skilled groups were more likely to be concerned with barriers such as environmental exposures, weather elements, and terrains. The difference in concerns about these barriers among groups was also more pronounced.

\section{Discussion}

\subsection{Discussion of the results}

Our study examined the behavior and priorities of women cyclists across multiple areas in the US and Canada. We explored respondents' behavior by skill group as defined by Geller (2006). The results indicate that women cyclists identify bicycle facilities and bicycle culture in their communities and workplace as motivators for the choice to cycle. Although a majority of the participants cycled for utilitarian and non-utilitarian purposes, the patterns substantially differ among cyclists of the four skill groups. Lower skill groups, such as No Way No How and Interested but Concerned were less likely to cycle for utilitarian purposes. However, many Interested but Concerned cyclists cycled for non-utilitarian purposes. As the name indicates, the Strong and Fearless group did not have particular concerns about the surrounding environment; however, they were concerned with the availability and quality of cycling facilities and building a cycling culture. In general, these findings are consistent with those from previous studies (Dill \& McNeil, 2013, 2016).

Our results show that while we oversampled experienced cyclists who expressed that they were likely to cycle on streets with either mixed traffic or bike lanes, a majority of our respondents reported that their ideal infrastructure was separated bike lanes, trails, or other types of protected infrastructure (i.e., facilities that are physically separated from vehicular traffic). These results, coupled with other findings presented in the previous section, suggest that even experienced and skilled women cyclists were concerned with safety and interactions with motorized traffic. This concern may also be true for male cyclists, a topic that deserves more exploration in the future.

We also found that most women cyclists in our sample were choice riders. This finding is likely due to our sampling method as we distributed the survey through social media and bicycle advocacy groups. However, we believe this reflects the nature of women cycling, as noted in Singleton and Goddard (2016). In a study based on a probability sample in Oregon, they found that most women cyclists were by choice, not by necessity. Nonetheless, even for these choice cyclists, practicality was still important. 
This means that cycling has to be a convenient and reliable transport option for women to start cycling and/or to maintain their cycling amount.

This study found that most women cyclists were environmentally focused, given that sustainability was frequently reported as an important motivation for them to cycle. This could be a potential marketing message to encourage more women to cycle in the future. Additionally, having a sense of purpose (i.e., cycling to protect the environment) would increase cyclists' eudaimonic well-being (Nordbakke \& Schwanen, 2014; Singleton, 2017), as they are practicing a good deed on a daily basis.

Another highly reported factor for cycling is joy. A majority of high skill cyclist groups perceived cycling as providing joy, while half of the Interested but Concerned group reported this reason. Nonetheless, this finding is consistent with the literature on satisfaction and happiness, where cyclists were found to be the happiest among all transport users (Morris \& Guerra, 2014; Páez \& Whalen, 2010; Smith, 2017). Joy is another aspect of well-being, namely hedonic well-being, and has been promoted widely thanks to its positive effect on physical and mental health. Cycling, as well as other forms of exercising, releases endorphins, hormones that interact with receptors in human brains to reduce the perception of pain. Keeping one's fitness and reducing risks for cardiovascular diseases are also good motivations for cycling, as reported by respondents in the "health" factors.

All groups expressed concerns about a cycling culture within their community and/or workplace and schools. This is reasonable given that cycling remains uncommon in many smaller cities. Perhaps gender bias still plays a role that discriminates women who cycle. We were not able to compare with men cyclists, although future studies with other groups of cyclists would shed light on this issue.

\subsection{Strengths, limitations, and future research}

Our study has several strengths in sampling and methods. It is among the first studies to focus specifically on women cyclists and identify key motivators and barriers to encourage or maintain cycling rates. The survey has good geographical coverage and includes cyclists from most US states and Canadian provinces. Additionally, this study is among a few studies that segmented cyclists' motivators and barriers by skill level. We applied machine learning models to determine the most important factors that influence the cycling behavior for utilitarian and non-utilitarian purposes. The cross validation results showed that the models were robust.

This study makes several contributions to transportation research and practice. Planners may address the needs of women cyclists in bicycle plans and consider education and encouragement strategies identified in this study as a means to promote cycling among women. Second, the difference in perceptions and behaviors of cyclists among the skill groups indicates the need to consider a market segmentation approach based on cyclists' comfort or skill level. Third, our results show that the Interested but Concerned group is more likely to cycle for non-utilitarian purposes. Participation in recreational cycling may help to build the cycling skills and comfort needed to shift to utilitarian cycling. This group is most likely to diversify their trip purposes when changes in the transportation network and communities happen. At the same time, the No Way No How group would benefit from investment in education and infrastructure to change their attitude and perceptions. Community programs and activities, such as Bike to Work day, as well as participating in recreational cycling may help them gradually adapt to cycling and ultimately switch to cycling for transportation purposes.

Our approach suffers from limitations related to study design, sampling, and methods. While the study was designed to be easily applied in planning practice, the survey was short to reduce response burden (which resulted in a fairly high response rate), and did not cover many items that may affect cycling behavior, for example, household composition, life cycle, income, employment status, cycling frequency, number of trips, distance or duration, and travel patterns by other modes, among others. Second, given that the survey was distributed among groups of proactive cyclists, we oversampled a large number of high-skill women cyclists and those who were highly educated. Although studies show that cyclists 
tend to have higher educational attainment and slightly higher income than non-cyclists (Moudon et al., 2005), this overrepresentation of cyclists with a graduate or college degree would certainly affect our results. Nevertheless, while these women are not representative of the general population, their voices are important to hear as they are likely out riding in current conditions, possess valuable knowledge about cycling in our communities, and can help encourage those in the Interested but Concerned group onto their bicycles.

It is noteworthy that most cyclists in our sample did not have school-age children. People without children usually have more flexible schedules compared to those with children. With traditional roles in a family, women usually perform more housework, running errands, and do most childcare duties. As such, studies found that women, especially those with children, tend to have higher trip complexity and more trip chaining (McGuckin et al., 2005). It is unknown whether most women cyclists, in general, do not have school-age children, or it is unique to our sample. A study in Oregon found that women with two children were more likely to cycle, but those with children aged 6-11 were not (Singleton \& Goddard, 2016). A UK-based study found that women with children were less likely to commute by bicycle (Grudgings et al., 2018). As such, more studies are needed to explore the effect of household composition on cycling among women and to identify potential policies to encourage women with younger children to cycle.

These limitations suggest several directions for future research. Adding more trip-level variables (e.g., travel patterns, cycling frequency) would be beneficial to understand the relationship between cycling and perceptions of safety and comfort. Further, it would be useful to study women who do not cycle to investigate opportunities for behavioral change. Finally, a comparison between women and men cyclists would be helpful to understand any gender differences

\section{Conclusions}

This study examines the behavior and perceptions of women cyclists in North America through two dimensions: trip purpose and skill level. We found that practicality, health, and sustainability were highly reported among all skill groups and were the most common motivations to cycle for utilitarian and nonutilitarian purposes. Joy and affordability were reported more often in high skill groups. Very few cyclists cycled by choice. Cycling culture and infrastructure were among the common barriers that discouraged cyclists to cycle more frequently and more comfortably.

Our work has several implications for planning practice. Planners should consider motivators and barriers identified in this study to encourage more women cyclists and thus create a cycling culture by integrating these factors into bicycle plans and street designs. Planners may tailor their strategies to target different groups of cyclists by skill; for example, encouraging the Interested but Concerned cyclists who mostly cycle for recreation on off-street facilities to participate in utilitarian trips. By increasing investment in bicycle infrastructure and other encouragement policies, planners may shift many cyclists in this group to increase cycling rates for all purposes. Changing culture and education may also have spillover effects to increase cycling adoption in the No Way No How cyclists. Future research could replicate this study for a wider range of women cyclists (i.e., more low skill cyclists and non-cyclists), as well as expanding our work by surveying men who cycle. Expanding the participant sample would allow for comparing the patterns of cycling among men and women as well as those who cycle and those who do not.

\section{Acknowledgements}

We thank the Association of Bicycle and Pedestrian Professionals, Kit Keller, and Candice Dias for coordinating the Women Cycling Project, and Noreen McDonald for guiding an early exploratory analysis on this dataset. We also thank all the participants for making this study possible. 


\section{References}

Abasahl, F., Kelarestaghi, K. B., \& Ermagun, A. (2018). Gender gap generators for bicycle mode choice in Baltimore college campuses. Travel Behavior and Society, 11, 78-85. https://doi.org/10.1016/j. tbs.2018.01.002

Akar, G., Fischer, N., \& Namgung, M. (2013). Bicycling choice and gender case study: The Ohio State University. International Journal of Sustainable Transportation, 7(5), 347-365. https://doi.org/10.10 80/15568318.2012.673694

Aldred, R. (2016). Cycling near misses: Their frequency, impact, and prevention. Transportation Research Part A: Policy and Practice, 90, 69-83. https://doi.org/10.1016/j.tra.2016.04.016

Antoniou, C., \& Koutsopoulos, H. (2006). Estimation of traffic dynamics models with machinelearning methods. Transportation Research Record: Journal of the Transportation Research Board, 1965, 103-111. https://doi.org/10.3141/1965-11

Arentze, T. A., \& Timmermans, H. J. P. (2004). A learning-based transportation-oriented simulation system. Transportation Research Part B: Methodological, 38(7), 613-633. https://doi.org/10.1016/j. trb.2002.10.001

Bonham, J., \& Wilson, A. (2012). Bicycling and the life course: The start-stop-start experiences of women cycling. International Journal of Sustainable Transportation, 6(4), 195-213. https://doi.org/1 $0.1080 / 15568318.2011 .585219$

Breiman, L. (1996). Bagging predictors. Machine Learning, 24(2), 123-140. doi org/10.1023/ A: 1018054314350

Breiman, L. (2001). Random forests. Machine Learning, 45(1), 5-32. https:/doi. org/10.1023/A:1010933404324

Bühlmann, P., \& Hothorn, T. (2007). Boosting algorithms: Regularization, prediction and model fitting. Statistical Science, 22(4), 477-505. https://doi.org/10.1214/07-STS242

Damant-Sirois, G., \& El-Geneidy, A. M. (2015). Who cycles more? Determining cycling frequency through a segmentation approach in Montreal, Canada. Transportation Research Part A: Policy and Practice, 77, 113-125. https://doi.org/10.1016/j.tra.2015.03.028

Dill, J. (2009a). Bicycling for transportation and health: The role of infrastructure. Journal of Public Health Policy, 30(1), S95-S110.

Dill, J. (2009b). Comfort + convenience $=$ more women biking. The League of American Bicyclists Webinar. Retrieved from https://bikeleague.org/sites/default/files/Dill\%20LAB\%20women\%20 on\%20a\%20roll\%20webinar.pdf on May 8, 2019.

Dill, J., \& McNeil, N. (2013). Four types of cyclists? Examination of typology for better understanding of bicycling behavior and potential. Transportation Research Record, 2387(1), 129-138. https://doi. org/10.3141/2387-15

Dill, J., \& McNeil, N. (2016). Revisiting the four types of cyclists. Transportation Research Record: Journal of the Transportation Research Board, 2587, 90-99. https://doi.org/10.3141/2587-11

Ding, A., Zhao, X., \& Jiao, L. (2002). Traffic flow time series prediction based on statistics learning theory. In Proceedings of the IEEE 5th International Conference on Intelligent Transportation Systems (pp. 727-730). Retrieved from https://doi.org/10.1109/ITSC.2002.1041308

Ding, C., Cao, X., \& Næss, P. (2018). Applying gradient boosting decision trees to examine non-linear effects of the built environment on driving distance in Oslo. Transportation Research Part A: Policy and Practice, 110, 107-117. https://doi.org/10.1016/j.tra.2018.02.009

Emond, C., Tang, W., \& Handy, S. (2009). Explaining gender difference in bicycling behavior. Transportation Research Record: Journal of the Transportation Research Board, 2125, 16-25. https://doi. 
org/10.3141/2125-03

Garrard, J. (2003). Healthy revolutions: Promoting cycling among women. Health Promotion Journal of Australia, 14(3), 213-215. https://doi.org/10.1071/he03213

Garrard, J., Handy, S., \& Dill, J. (2012). Women and cycling. In City Cycling (pp. 211-234). Cambridge, MA: MIT Press. Retrieved from http://www.jstor.org/stable/j.ctt5hhjxr.14

Garrard, J., Rose, G., \& Lo, S. K. (2008). Promoting transportation cycling for women: The role of bicycle infrastructure. Preventive Medicine, 46(1), 55-59. https://doi.org/10.1016/j.ypmed.2007.07.010

Geller, R. (2006). Four types of transportation cyclists. Retrieved from https://www.portlandoregon. gov/transportation/article/158497

Gong, L., Kanamori, R., \& Yamamoto, T. (2017). Data selection in machine learning for identifying trip purposes and travel modes from longitudinal GPS data collection lasting for seasons. Travel Behavior and Society. https://doi.org/10.1016/j.tbs.2017.03.004

Gong, L., Morikawa, T., Yamamoto, T., \& Sato, H. (2014). Deriving personal trip data from GPS data: A literature review on the existing methodologies. Procedia - Social and Behavioral Sciences, 138, 557-565. https://doi.org/10.1016/j.sbspro.2014.07.239

Grudgings, N., Hagen-Zanker, A., Hughes, S., Gatersleben, B., Woodall, M., \& Bryans, W. (2018). Why don't more women cycle? An analysis of female and male commuter cycling mode-share in England and Wales. Journal of Transport \& Health. https://doi.org/10.1016/j.jth.2018.07.004

Hastie, T., Tibshirani, R., \& Friedman, J. (2009). The Elements of Statistical Learning: Data Mining, Inference, and Prediction, Second Edition (2nd ed.). New York: Springer-Verlag.

Heesch, K. C., Sahlqvist, S., \& Garrard, J. (2012). Gender differences in recreational and transport cycling: A cross-sectional mixed-methods comparison of cycling patterns, motivators, and constraints. International Journal of Behavioral Nutrition and Physical Activity, 9(1), 106.

Jahangiri, A., \& Rakha, H. A. (2015). Applying machine learning techniques to transportation mode recognition using mobile phone sensor data. IEEE Transactions on Intelligent Transportation Systems, 16(5), 2406-2417. https://doi.org/10.1109/TITS.2015.2405759

James, G., Witten, D., Hastie, T., \& Tibshirani, R. (2014). An Introduction to statistical learning: With Applications in R. New York: Springer.

Ma, L., Dill, J., \& Mohr, C. (2014). The objective versus the perceived environment: What matters for bicycling? Transportation, 41(6), 1135-1152. https://doi.org/10.1007/s11116-014-9520-y

McGuckin, N., Zmud, J., \& Nakamoto, Y. (2005). Trip-chaining trends in the United States: Understanding travel behavior for policy making. Transportation Research Record: Journal of the Transportation Research Board, 1917, 199-204. https://doi.org/10.3141/1917-22

Morris, E. A., \& Guerra, E. (2014). Mood and mode: Does how we travel affect how we feel? Transportation, 42(1), 25-43. https://doi.org/10.1007/s11116-014-9521-x

Moudon, A. V., Lee, C., Cheadle, A. D., Collier, C. W., Johnson, D., Schmid, T. L., \& Weather, R. D. (2005). Cycling and the built environment, a US perspective. Transportation Research Part D: Transport and Environment, 10(3), 245-261. https://doi.org/10.1016/j.trd.2005.04.001

Nordbakke, S., \& Schwanen, T. (2014). Well-being and mobility: A theoretical framework and literature review focusing on older people. Mobilities, 9(1), 104-129. https://doi.org/10.1080/1745010 1.2013 .784542

Páez, A., \& Whalen, K. (2010). Enjoyment of commute: A comparison of different transportation modes. Transportation Research Part A: Policy and Practice, 44(7), 537-549. https://doi.org/10.1016/j. tra.2010.04.003

Pucher, J., Buehler, R., \& Seinen, M. (2011). Bicycling renaissance in North America? An update and re-appraisal of cycling trends and policies. Transportation Research Part A: Policy and Practice, 45(6), 451-475. https://doi.org/10.1016/j.tra.2011.03.001 
Sibley, A. (2010). Women's cycling survey: Analysis of results. University of North Carolina Greensboro. Retrieved from http://womencyclingproject.info/wp-content/uploads/2014/04/womens_cycling survey_091420.pdf

Singleton, P. A. (2017). Exploring the positive utility of travel and mode choice. Retrieved from https:// rosap.ntl.bts.gov/view/dot/35399

Singleton, P. A., \& Goddard, T. (2016). Cycling by choice or necessity? Transportation Research Record: Journal of the Transportation Research Board, 2598, 110-118. https://doi.org/10.3141/2598-13

Smith, O. (2017). Commute well-being differences by mode: Evidence from Portland, Oregon, USA. Journal of Transport \& Health, 4(Supplement C), 246-254. https://doi.org/10.1016/j. jth.2016.08.005

Teschke, K., Chinn, A., \& Brauer, M. (2017). Proximity to four bikeway types and neighborhoodlevel cycling mode share of male and female commuters. Journal of Transport and Land Use, 10(1) 695-713. https://doi.org/10.5198/jtlu.2017.943

Varian, H. R. (2014). Big data: New tricks for econometrics. Journal of Economic Perspectives, 28(2), 3-28. https://doi.org/10.1257/jep.28.2.3

Xing, Y., Handy, S. L., \& Mokhtarian, P. L. (2010). Factors associated with proportions and miles of bicycling for transportation and recreation in six small US cities. Transportation Research Part D: Transport and Environment, 15(2), 73-81. https://doi.org/10.1016/j.trd.2009.09.004 